\title{
Metformin inhibits proliferation of human keratinocytes through a mechanism associated with activation of the MAPK signaling pathway
}

\author{
WEINING LI ${ }^{1,2}$, WEIYUAN MA ${ }^{1}$, HUA ZHONG $^{1}$, WENBIN LIU $^{1}$ and QING SUN ${ }^{1}$ \\ ${ }^{1}$ Department of Dermatology, Qilu Hospital, Shandong University; ${ }^{2}$ Department of Dermatology, \\ Affiliated Hospital of Shandong University of Traditional Chinese Medicine, Jinan, Shandong 250012, P.R. China
}

Received July 4, 2013; Accepted November 5, 2013

DOI: $10.3892 /$ etm.2013.1416

\begin{abstract}
In the present study, the effects of metformin on the proliferation of human immortalized keratinocytes (HaCaTs) and the underlying mechanisms were investigated. $\mathrm{HaCaT}$ cells in the logarithmic growth phase were treated with $50 \mathrm{mM}$ metformin for 24, 48 and $72 \mathrm{~h}$. Cell morphology after $24 \mathrm{~h}$ of treatment was observed under a microscope. Cell proliferation was detected using a colorimetric cell proliferation and cytotoxicity assay kit. Western blot analyses were performed to detect the protein phosphorylation levels of adenosine monophosphate-activated protein kinase (AMPK) and extracellular signal-related kinase 1/2 (ERK1/2). Metformin treatment resulted in morphological changes of the $\mathrm{HaCaT}$ cells. The survival rates of $\mathrm{HaCaT}$ cells treated with metformin were $36.18,12.70$ and $10.12 \%$ at 24,48 and $72 \mathrm{~h}$, respectively. As the treatment time extended, the survival rates of HaCaT cells decreased. Western blot analysis results showed that the mean level of phosphorylated (p)-AMPK in the HaCaT cells without metformin treatment was $2.856 \pm 0.323$. However, the mean p-AMPK level following metformin treatment for $24 \mathrm{~h}$ increased to $5.198 \pm 0.625$, indicating a significant difference between these two groups $(\mathrm{P}<0.05)$. The mean absorbance ratio of p-ERK1/2 was $7.550 \pm 1.087$ for the untreated cells, but the levels in cells following metformin treatment for $24 \mathrm{~h}$ increased to $10.430 \pm 1.217$, indicating a significant difference between the two groups $(\mathrm{P}<0.05)$. In conclusion, metformin treatment upregulated the levels of p-AMPK and p-ERK1/2 in $\mathrm{HaCaT}$ cells, and significantly inhibited $\mathrm{HaCaT}$ cell prolifera-
\end{abstract}

Correspondence to: Dr Qing Sun, Department of Dermatology, Qilu Hospital, Shandong University, 107 West Wenhua Road, Jinan, Shandong 250012, P.R. China

E-mail: liweining1981@163.com

Key words: metformin, HaCaT cells, proliferation, psoriasis, adenosine monophosphate-activated protein kinase, extracellular signal-related kinase $1 / 2$ tion in vitro by a mechanism associated with activation of the mitogen-activated protein kinase signaling pathway.

\section{Introduction}

Psoriasis is a chronic inflammatory skin disease with genetic predisposition (1-4), characterized by the hyperproliferation and abnormal differentiation of epidermal keratinocytes (5). Inhibition of the excessive proliferation of keratinocytes is the main treatment method of psoriasis (6). HaCaTs are immortalized cell lines derived from keratinocytes in normal adult skin and the excessive proliferation of these keratinocytes results in psoriatic lesions (7). Therefore, HaCaT cells have been widely used as an in vitro model for the study of anti-psoriasis agents $(8,9)$.

Metformin is an insulin sensitizer, it is the first-line treatment method for type II diabetes and was recommended by the American Diabetes Association in 2012 for its hypoglycemic effects and ability to reduce cardiovascular morbidity and mortality. In addition, metformin rarely causes lactic acidosis $(10,11)$. Previous studies have shown that metformin inhibits cell growth and proliferation of a number of types of cancer, including liver, colon and prostate cancer (12-14). However, whether metformin inhibits the proliferation of $\mathrm{HaCaT}$ cells has, to the best of our knowledge, not been studied.

The mitogen-activated protein kinase (MAPK) signaling pathway is important for the proliferation of HaCaT cells. It has been reported that metformin activates adenosine monophosphate-activated protein kinase (AMPK) in breast cancer MCF-7 cells, inhibits the mTOR signaling pathway to reduce protein translation initiation and decreases cell proliferation (15). Following metformin treatment in ovarian cancer cells, the AMPK signaling pathway is activated and phosphorylated (p)-AMPK protein expression is increased. This results in the inhibition of proliferation-associated protein molecule synthesis and thus suppression of ovarian cancer cell proliferation (16). Metformin also activates AMPK to reduce proliferative signaling in tumor cells and provide direct anti-tumor effects (17).

In the present study, the effects of metformin on $\mathrm{HaCaT}$ cell proliferation and the regulatory protein expression were evaluated and the molecular mechanisms of action of metformin in the treatment of psoriasis were investigated. 


\section{Materials and methods}

Cells and reagents. The $\mathrm{HaCaT}$ cell line was purchased from the American Type Culture Collection (Manassas, VA, USA). Metformin hydrochloride was purchased from Sigma-Aldrich (St. Louis, MO, USA). The cell proliferation and cytotoxicity assay kit (Cell Counting Kit-8; CCK-8) was purchased from Dojindo (Kunamoto, Japan). p-AMPK $\alpha 1$ and p-MAPK 1/2 antibodies were purchased from Abcam (Cambridge, MA, USA). The quantitative automatic microplate reader (model no., 2010) was purchased from Anthos Labtec Co., Ltd. (Salzburg Austria). The study was approved by the ethics committee of Shandong University (Jinan, China).

Metformin treatment. HaCaT cells were cultured in Dulbecco's modified Eagle's medium (DMEM) with $10 \%$ fetal bovine serum, $100 \mathrm{U} / \mathrm{ml}$ penicillin and $100 \mu \mathrm{g} / \mathrm{ml}$ streptomycin at $37^{\circ} \mathrm{C}$ in a $5 \% \mathrm{CO}_{2}$ humidified and sterile environment. $\mathrm{HaCaT}$ cells during the logarithmic growth phase were collected

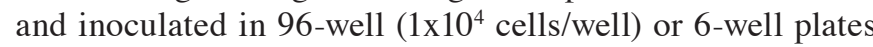
$\left(3 \times 10^{5}\right.$ cells/well). Two groups, specifically, control (without metformin) and metformin (50 $\mathrm{mM}$ metformin) were established. After $24 \mathrm{~h}$ of inoculation, the culture medium in the metformin group was replaced with DMEM containing metformin to maintain the metformin concentration at $50 \mathrm{mM}$. An equal volume of phosphate-buffered saline (PBS) was added to the control group.

Cell morphology observation. Following $24 \mathrm{~h}$ of $50 \mathrm{mM}$ metformin treatment, the morphology of $\mathrm{HaCaT}$ cells was observed under an inverted microscope (Olympus BX-51; Olympus optical Co., Ltd., Tokyo, Japan).

CCK-8 assay. Following 3-5 stable passages, HaCaT cells in the logarithmic phase were inoculated in 96-well plates. Cell culture medium (100 $\mu$ l DMEM; Invitrogen, Carlsbad, CA, USA) and $100 \mu 1$ metformin were added to the center of 60 wells. Following cross mixing, cells were cultured at $37^{\circ} \mathrm{C}$ in $5 \% \mathrm{CO}_{2}$ for 24,48 and $72 \mathrm{~h}$. CCK- 8 solution was added and the optical density (OD) values were detected at $450 \mathrm{~nm}$ using a quantitative automatic microplate reader (model no. 2010; Anthos Labtec Co., Ltd.). Cell survival rates at the various treatment times were calculated and the cell survival curve was drawn. The cell survival rate (\%) was calculated using the following formula: $\left(\mathrm{OD}_{\text {metformin }}-\mathrm{OD}_{\text {control }}\right) /\left(\mathrm{O}_{\text {Dcontrol }}-\mathrm{OD}_{\text {metformin }}\right) \times 100$.

Western blot analyses. Total protein was extracted from each sample and antibody incubation was performed according to the instructions of the manufacturer of the one-step rapid WB kit (rabbit; Shanghai Biological Engineering Co., Ltd., Shanghai, China) using antibodies against p-AMPK (1:200) and p-extracellular signal-regulated kinase (ERK1/2; 1:250). The ultra-sensitive enhanced chemiluminescence kit (Biyuntian Biotechnology Institute, Beijing, China) was used for color development. The membrane was incubated with the ECL Plus A and Plus B reagents for 2 min at room temperature. The membrane was developed in the dark. The developed films were scanned using the AlphaImager gel imaging systems (AlphaImager, Santa Clara, CA, USA). The western blot images were then analyzed using Quantity One software (Bio-Rad Laboratories, Hercules, CA, USA). $\beta$-actin was used as an internal control. The relative absorbance ratios of $\mathrm{p}$-AMPK to $\beta$-actin and p-ERK1/2 to $\beta$-actin were defined as the relative values of $\mathrm{p}$-AMPK and p-ERK1/2, respectively.

Statistical analyses. All experimental data are presented as the mean \pm standard deviation. SPSS statistical software (v13.0; SPSS, Inc., Chicago, IL, USA) was used for analysis. One-way analysis of variance was used for mean comparisons. $\mathrm{P}<0.05$ was considered to indicate a statistically significant difference.

\section{Results}

Effect of metformin on HaCaT cells. To determine the effects of metformin treatment on the morphology of HaCaT cells, cells were observed under an inverted microscope after $24 \mathrm{~h}$ of treatment. The untreated $\mathrm{HaCaT}$ cells were in adherent growth and arranged in cobblestone and mosaic shapes. Cells were flat and polygonal with a clear boundary, abundant cytoplasm, and a round or oval nucleus (Fig. 1A). The cells in the metformin group were treated with $50 \mathrm{mM}$ metformin for $24 \mathrm{~h}$. The sizes of treated cells were slightly smaller compared with those of the untreated cells (Fig. 1B). The sizes of cell granules and vacuoles were increased and a number of cells had been killed by the treatment (Fig. 1B).

To investigate cell proliferation following metformin treatment, the CCK-8 cell viability assay was performed. As shown in Fig. 2, the survival fractions of $\mathrm{HaCaT}$ cells treated with metformin were $36.18,12.70$ and $10.12 \%$ at 24,48 and $72 \mathrm{~h}$, respectively, and were significantly lower compared with those of the untreated control $(\mathrm{P}<0.05)$. With extended metformin treatment time, $\mathrm{HaCaT}$ cell survival rates gradually decreased.

Collectively, these results suggest that metformin induces changes in $\mathrm{HaCaT}$ cell morphology and inhibits $\mathrm{HaCaT}$ cell proliferation in vitro.

Effect of metformin on AMPK and ERK1/2 protein expression in HaCaT cells. To determine whether the MAPK signaling pathway was activated by metformin, the levels of p-AMPK and p-ERK1/2 were detected by western blot analysis. As shown in Fig. $3 \mathrm{~A}$ and $\mathrm{B}$, in the cells treated with PBS, the mean absorbance ratio of $\mathrm{p}$-AMPK relative to $\beta$-actin in $\mathrm{HaCaT}$ cells was $2.856 \pm 0.323$. However the mean absorbance ratio of the cells treated with metformin was $5.198 \pm 0.625$. The expression levels of p-AMPK between these two groups were identified to be significantly different $(\mathrm{P}<0.05$; Fig. 3$)$.

As shown in Fig. 3A and $\mathrm{C}$, the mean absorbance ratio of p-ERK1/2 relative to $\beta$-actin in the untreated $\mathrm{HaCaT}$ cells was $7.550 \pm 1.087$, but the ratio in the cells treated with metformin was $10.430 \pm 1.217$, with a significant difference between the two groups $(\mathrm{P}<0.05)$. These results indicated that the above proteins were activated following metformin treatment for $24 \mathrm{~h}$. After $24 \mathrm{~h}$ of metformin treatment, the expression levels of p-AMPK and p-ERK1/2 markedly increased. This suggests that AMPK and ERK1/2 proteins were phosphorylated due to metformin treatment. Therefore, metformin inhibited $\mathrm{HaCaT}$ cell proliferation, possibly via a mechanism associated with the activation of the MAPK signaling pathway. 

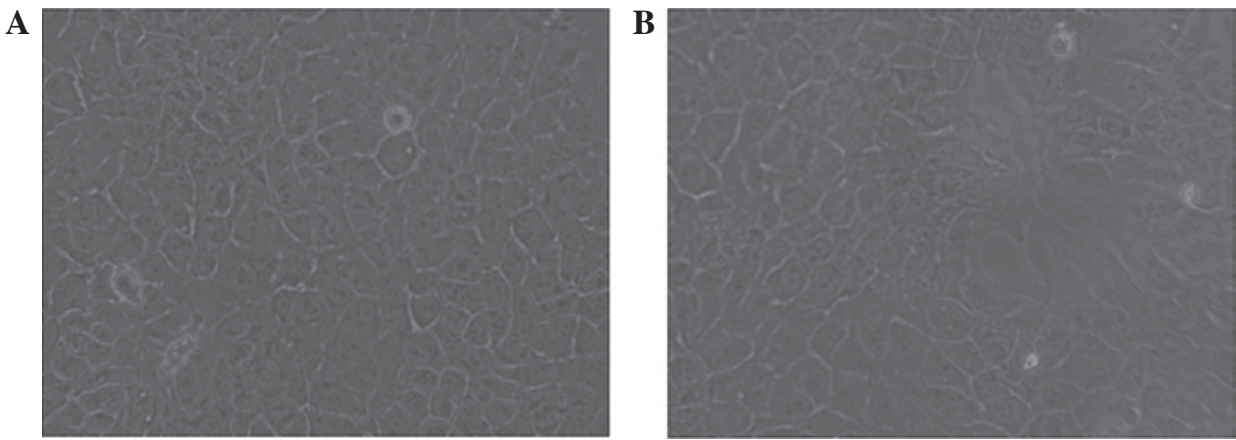

Figure 1. Morphology analysis of HaCaT cells following metformin treatment. Cells were observed under an inverted microscope (magnification, x200). (A) Untreated HaCaT cells and (B) HaCaT cells treated with $50 \mathrm{mM}$ metformin for $24 \mathrm{~h}$. HaCat, human immortalized keratinocytes.

A

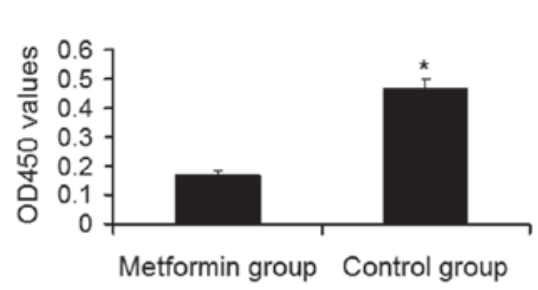

C

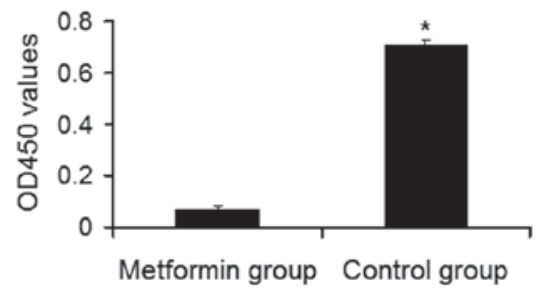

B

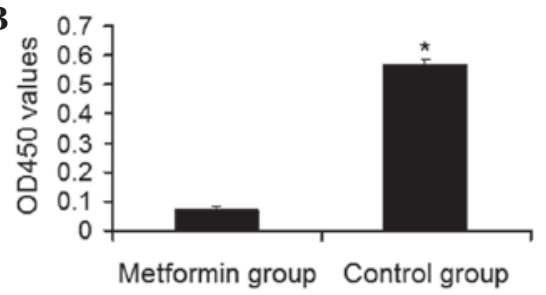

D

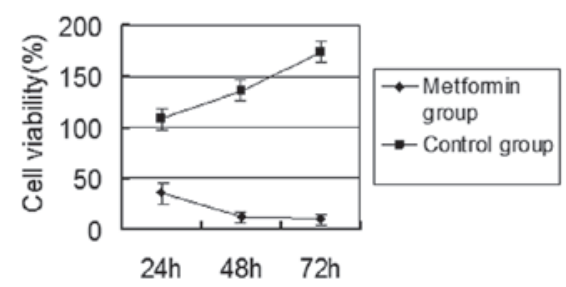

Figure 2. Cell proliferation analysis of HaCaT cells following metformin treatment. After treatment with $50 \mathrm{mM}$ metformin for 24,48 and $72 \mathrm{~h}$, cell viability was detected by a CCK- 8 kit. The OD values at $450 \mathrm{~nm}$ of each group were measured and the cell viability was calculated. OD value at $450 \mathrm{~nm}$ of each group at (A) 24,(B) 48 and (C) $72 \mathrm{~h}$. (D) Cell viability of each group at 24, 48 and $72 \mathrm{~h}$ following metformin treatment. Cell survival rate (\%) was calculated using the following formula: $\left(\mathrm{OD}_{\text {metformin }}-\mathrm{OD}_{\text {control }}\right) /\left(\mathrm{OD}_{\text {control }}-\mathrm{OD}_{\text {metformin }}\right) \times 100$. Experiments were conducted three times and data are expressed as the mean \pm standard deviation. ${ }^{*} \mathrm{P}<0.05$, vs. metformin group. HaCat, human immortalized keratinocytes; OD, optical density; CCK-8, cell proliferation and cytotoxicity assay kit-8.

A

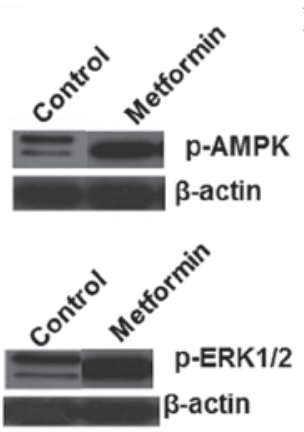

B

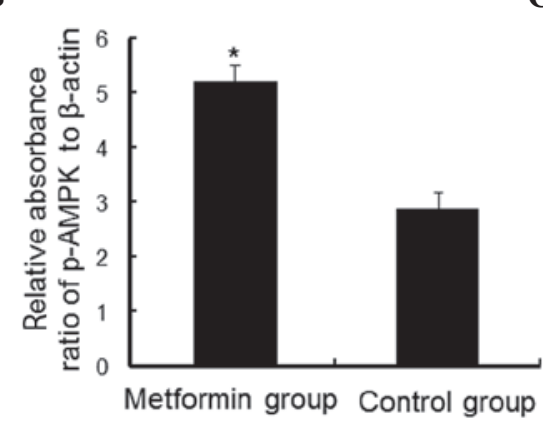

C

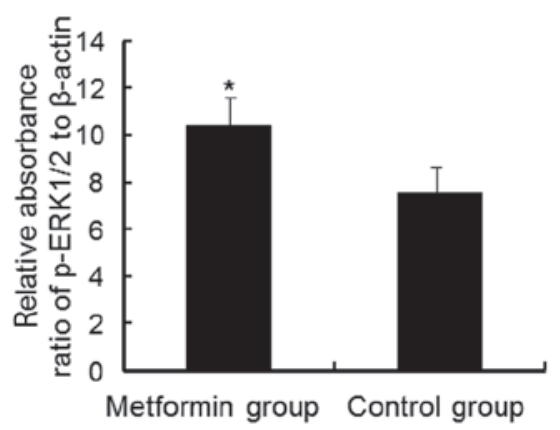

Figure 3. Analysis of p-AMPK and p-ERK1/2 expression in HaCaT cells following metformin treatment. Expression of p-AMPK and p-ERK1/2 in HaCaT cells was evaluated by western blot analysis $24 \mathrm{~h}$ after metformin treatment. $\beta$-actin was used as the internal control. (A) Western blot analysis results of p-AMPK and p-ERK1/2 expression levels and the relative absorbance ratio of (B) p-AMPK to $\beta$-actin of each group and (C) p-ERK1/2 to $\beta$-actin of each group. Data are presented the mean \pm standard deviation of three independent experiments. " $\mathrm{P}<0.05$, vs. the control group. p, phosphorylated; HaCat, human immortalized keratinocytes; AMPK, adenosine monophosphate-activated protein kinase; ERK1/2, extracellular signal-related kinase 1/2.

\section{Discussion}

In the present study, $\mathrm{HaCaT}$ cells were used as an in vitro model of psoriatic keratinocyte proliferation to analyze the effects of metformin on $\mathrm{HaCaT}$ cell proliferation and investigate the possible mechanisms of its treatment for psoriasis. The CCK-8 assay showed that metformin significantly inhibited the proliferation of $\mathrm{HaCaT}$ cells in vitro. Western blot analysis results suggested that metformin stimulated AMPK and ERK1/2 phosphorylation in $\mathrm{HaCaT}$ cells. These observations indicate 
that metformin activates AMPK in the ERK1/2 signaling pathway and regulates its downstream gene expression to inhibit cell proliferation. In addition, following metformin treatment, the levels of p-AMPK protein were significantly increased.

ERK1/2 is an important signaling pathway in the MAPK family that regulates cell growth and differentiation. The ERK1/2 signaling pathway is closely associated with psoriasis, but whether there is phosphorylation of ERK1/2 in psoriasis lesions remains controversial. ERK1/2 activation is dependent upon stimulus intensity, thus the intensity of ERK activation is likely to affect the response (18). It has been previously reported that the ERK1/2 signaling pathway exhibits dual effects in promoting $\mathrm{HaCaT}$ cell proliferation through epidermal growth factors whose effects on $\mathrm{HaCaT}$ cell proliferation and activation are associated with ERK signal intensity (19). However, overactivation of the ERK signal inhibits cell proliferation. For example, Pumiglia et al (20) reported that activation of ERK inhibits CDK activity and induces cell cycle arrest through induction of the CDK inhibitor $\mathrm{p} 21^{\mathrm{Cip} / \mathrm{WAF} 1}$. Wang et al (21) also observed that persistent activation of ERK lead to cell cycle arrest. Tang et al (22) showed that ERK activation partially contributed to $\mathrm{p} 21 \mathrm{Cip1/WAF1}$ induction. In the present study, we found that after metformin treatment, $\mathrm{HaCaT}$ cell proliferation was inhibited while p-ERK was upregulated. Therefore we suggest that the inhibition of metformin on $\mathrm{HaCaT}$ cells is mediated by ERK activation.

In conclusion, the MAPK signal transduction pathway is important in mammalian cells. In the present study, metformin enhanced the expression of p-ERK1/2, suggesting that the mechanism by which metformin inhibits cell proliferation may be associated with activation of the MAPK signaling pathway. However, the mechanisms associated with the transduction of stimulus signals via the ERK1/2 signaling pathway and whether other signaling pathways are involved, requires further study.

\section{Acknowledgements}

This study was supported by the National Natural Science Foundation of China (grant no. 81071291).

\section{References}

1. Vestergaard C, Deleuran M, Gesser B and Grønhøj Larsen C: Expression of the T-helper 2-specific chemokine receptor CCR4 on CCR10-positive lymphocytes in atopic dermatitis skin but not in psoriasis skin. Br J Dermatol 149: 457-463, 2003.

2. Bowcock AM and Krueger JG: Getting under the skin: the immunogenetics of psoriasis. Nat Rev Immunol 5: 699-711, 2005.

3. Bhalerao J and Bowcock AM: The genetics of psoriasis: a complex disorder of the skin and immune system. Hum Mol Genet 7: 1537-1545, 1998.
4. Bowcock AM and Cookson WO: The genetics of psoriasis, psoriatic arthritis and atopic dermatitis. Hum Mol Genet 13: R43-R55, 2004.

5. Abdou AG and Hanout HM: Evaluation of survivin and NF-kappaB in psoriasis, an immunohistochemical study. J Cutan Pathol 35: 445-451, 2008

6. Rahman M, Alam K, Ahmad MZ, et al: Classical to current approach for treatment of psoriasis: a review. Endocr Metab Immune Disord Drug Targets 12: 287-302, 2012.

7. Fusenig NE and Boukamp P: Multiple stages and genetic alterations in immortalization, malignant transformation, and tumor progression of human skin keratinocytes. Mol Carcinog 23: 144-158, 1998.

8. Stein M, Bernd A, Ramirez-Bosca A, Kippenberger S and Holzmann H: Measurement of anti-inflammatory effects of glucocorticoids on human keratinocytes in vitro. Comparison of normal human keratinocytes with the keratinocyte cell line HaCaT. Arzneimittelforschung 47: 1266-1270, 1997.

9. Müller K and Prinz H: Antipsoriatic anthrones with modulated redox properties. 4. Synthesis and biological activity of novel 9 , 10-dihydro-1,8-dihydroxy-9-oxo-2-anthracenecarboxylic and -hydroxamic acids. J Med Chem 40: 2780-2787, 1997.

10. Kahn BB, Alquier T, Caning D and Hardie DG: AMP-activated protein kinase: ancient energy gauge provides clues to modern understanding of metabolism. Cell Metab 1: 15-25, 2005.

11. Witters LA: The blooming of the French lilac. J Clin Invest 108: 1105-1107, 2001.

12. Zhang ZJ, Zheng ZJ, Shi R, Su Q, Jiang Q and Kip KE: Metformin for liver cancer prevention in patients with type 2 diabetes: a systematic review and meta-analysis. J Clin Endocrinol Metab 97: 2347-2353, 2012.

13. Buzzai M, Jones RG, Amaravadi RK, Lum JJ, DeBerardinis RJ, Zhao F, Viollet B and Thompson CB: Systemic treatment with the antidiabetic drug metformin selectively impairs p53-deficient tumor cell growth. Cancer Res 67: 6745-6752, 2007.

14. Ben Sahra I, Laurent K, Loubat A, et al: The antidiabetic drug metformin exerts an antitumoral effect in vitro and in vivo through a decrease of cyclin D1 level. Oncogene 27: 3576-3586, 2008.

15. Dowling RJ,Zakikhani M, Fantus IG, Pollak M and Sonenberg N: Metformin inhibits mammalian target of rapamycin-dependent translation initiation in breast cancer cells. Cancer Res 67: 10804-10812, 2007.

16. Rattan R, Giri S, Hattmann L and Shridhar V: Metformin attenuates ovarian cancer cell growth in an AMP-kinase dispensable manner. J Cell Mol Med 15: 166-178, 2011.

17. Zakikhani M, Dowling R, Fantus IG, Sonenberg N and Pollak M: Metformin is an AMP kinase-dependent growth inhibitor for breast cancer cells. Cancer Res 66: 10269-10273, 2006.

18. Bosch M, Gil J, Bachs O and Agell N: Calmodulin inhibitor W13 induces sustained activation of ERK2 and expression of p21(cip1). J Biol Chem 273: 22145-22150, 1998.

19. Wang H, Zeng Y, Ji Y and Xing F: Two-sided effect of ERK signal pathway on HaCaT cell proliferation induced by EGF. Basic \& Clinical Medicine 26: 471-475, 2006 (In Chinese).

20. Pumiglia KM and Decker SJ: Cell cycle arrest mediated by the MEK/mitogen-activated protein kinase pathway. Proc Natl Acad Sci USA 94: 448-452, 1997.

21. Wang Z, Zhang B, Wang M and Carr BI: Persistent ERK phosphorylation negatively regulates cAMP response element-binding (CREB) activity via recruitment of CREB-binding protein to pp90RSK. J Biol Chem 278: 11138-11144, 2003.

22. Tang D, Wu D, Hirao A, et al: ERK activation mediates cell cycle arrest and apoptosis after DNA damage independently of p53. J Biol Chem 277: 12710-12717, 2002. 